\title{
Objectively Measuring Signal Detectability, Contrast, Blur and Noise in Medical Images using Channelized Joint Observers
}

\author{
Bart Goossens, Hiêp Luong, Ljiljana Platiša and Wilfried Philips \\ Ghent University, Dept. of Telecommunications and Information Processing, TELIN-IPI-iMinds, \\ Sint-Pietersnieuwstraat 41, 9000 Gent, Belgium
}

\begin{abstract}
To improve imaging systems and image processing techniques, objective image quality assessment is essential. Model observers adopting a task-based quality assessment strategy by estimating signal detectability measures, have shown to be quite successful to this end. At the same time, costly and time-consuming human observer experiments can be avoided. However, optimizing images in terms of signal detectability alone, still allows a lot of freedom in terms of the imaging parameters. More specifically, fixing the signal detectability defines a manifold in the imaging parameter space on which different "possible" solutions reside. In this article, we present measures that can be used to distinguish these possible solutions from each other, in terms of image quality factors such as signal blur, noise and signal contrast. Our approach is based on an extended channelized joint observer (CJO) that simultaneously estimates the signal amplitude, scale and detectability. As an application, we use this technique to design $k$-space trajectories for MRI acquisition. Our technique allows to compare the different spiral trajectories in terms of blur, noise and contrast, even when the signal detectability is estimated to be equal.
\end{abstract}

Keywords: Channelized Hotelling observer, medical image quality assessment

\section{INTRODUCTION}

To optimize and improve medical image reconstruction techniques and display devices, objective quality assessment is essential. By objective approaches, time consuming and expensive experiments involving medical specialists can be avoided. In the past decades, model observers have been quite successful for objective image quality assessment in detection tasks. The model observer's detection performance, which serves as a predictor of a radiologist performing the same task, is then used to objectively compare the results of two (or multiple) image sets.

In recent medical image reconstruction techniques (e.g. MRI, CT, ...), often a lot of imaging parameters need to be determined. For example, in MRI, ${ }^{1}$ shorter acquisition techniques are preferred to avoid patient motion artifacts and to enable capturing dynamic processes (e.g. in cardiac examinations or angiography). One of these techniques, under-sampled $\mathrm{k}$-space acquisition based on optimized trajectories, has become very popular in the last decade. However, the design of such a trajectory is quite complicated: first, there are hardware and sequence constraints, and second, there are many trajectory parameters (and correspondingly, reconstruction parameters) to tune. The influence of a particular parameter choice on the diagnostic image quality is far from clear, and in literature many approaches revert to simple objective quality metrics, such as mean square error (MSE), or signal-to-noise-ratio (SNR), if a ground-truth reconstruction is available. The same trend occurs in CT reconstruction, where acquisition speed-ups can be obtained using limited projection views or helical scan trajectories. ${ }^{2}$ Again, CT reconstruction techniques need to be tuned to the corresponding acquisition schemes.

In medical imaging, task-based image quality optimization is much more useful than optimization based on global metrics such as MSE or SNR, because task-based measures are directly related to the way radiologists look at images: the image quality is determined by the ability to detect an object of interest (e.g. a lesion, tissue, tumor etc) to subsequently perform a diagnosis. However, when optimizing reconstruction techniques with many parameters in terms of a taskbased image quality criterion alone (such as area under the ROC - AUC), another problem arises: many combinations of parameter values lead to images with the same AUC value, even though the quality of these images in terms of noise, blur and contrast can differ significantly. Generally speaking, fixing the signal detectability defines a manifold in the imaging

Bart Goossens is a postdoctoral fellow of the Research Foundation Flanders (FWO), Belgium. 
parameter space on which different "possible" solutions reside. Extra quality measures are then needed to choose the "desired" solution on this manifold.

The approach that we use to tackle this problem in this paper is based on our recent research ${ }^{3}$ on channelized Hotelling observers designed for signal-known-statistically (SKS) detection tasks. SKS detection tasks are not only clinically more realistic than corresponding signal-known-exactly (SKE) detection tasks, but as we will show in this article, in terms of quality optimization, the statistical uncertainty with respect to the signal is also caused by the specific acquisition/reconstruction process with their parameters. In particular, our channelized joint observer (CJO) for SKS tasks, allows us to jointly estimate signal parameters (e.g., size, orientation and amplitude) together with the signal presence. Now, the estimation results can be used to directly obtain related image quality factors, such as blur and signal contrast.

The remainder of this paper is structured as follows: in Section 2, we give a brief overview of the channelized joint observer model. In Section 3, we discuss how the CJO can be used to assess blur, noise and contrast in medical images, while jointly estimating the signal detectability.

\section{OVERVIEW OF THE CHANNELIZED JOINT OBSERVER (CJO) MODEL}

For full details of the CJO model, we refer to Ref. ${ }^{3}$ Here, we give an overview of the model and discuss the aspects that are of importance for this paper. The task of the CJO is to detect a random parametric signal in the image. Let $\mathbf{x}$ denote a known parametric signal vector, let $\mathbf{b}$ and $\mathbf{y}$ denote a vectors of intensities of respectively the background image and the observed image, then the detection problem can be formulated as follows:

$$
\mathbf{y}= \begin{cases}\mathbf{b} & \left(H_{0}\right) \\ \mathbf{b}+\mathbf{x} & \left(H_{1}\right)\end{cases}
$$

The model observer decides whether a signal is present in the considered image $\left(H_{1}\right)$, or not $\left(H_{0}\right)$. This is done by comparing the decision test statistic $t$ to a given predefined threshold. ${ }^{3,4}$

The signal $\mathbf{x}$ is parametric, with a possibly unknown location and unknown shape parameters. One simple example is an elliptical Gaussian profile:

$$
[\mathbf{x}]_{\mathbf{q}}=a \exp \left(-\left(\mathbf{R}_{\vartheta} \mathbf{q}-\mathbf{p}\right)^{T} \mathbf{D}^{-1}\left(\mathbf{R}_{\vartheta} \mathbf{q}-\mathbf{p}\right)\right)
$$

where $a$ is the signal amplitude, $\mathbf{q}=\left[q_{x}, q_{y}\right]$ is a $2 \mathrm{D}$ vector denoting the spatial position, $\mathbf{p}$ is the position of the center of the signal and $\mathbf{D}=\left(\begin{array}{cc}2 b \cdot \sigma_{x}^{2} & 0 \\ 0 & 2 \cdot \sigma_{x}^{2}\end{array}\right)$ is a diagonal matrix with $b$ a fixed (known) constant and $\sigma_{x}$ an unknown scale parameter. $[\mathbf{x}]_{\mathbf{q}}$ denotes the $\left(q_{x}+\left(q_{y}-1\right) \sqrt{N}\right)$-th element of the vector $\mathbf{x}$, where $N$ is the number of pixels in the image (assuming a square image). Furthermore, $\mathbf{R}_{\vartheta}$ is a $2 \mathrm{D}$ rotation matrix:

$$
\mathbf{R}_{\vartheta}=\left(\begin{array}{cc}
\cos \vartheta & -\sin \vartheta \\
\sin \vartheta & \cos \vartheta
\end{array}\right)
$$

An elliptical Gaussian signal is depicted in Figure 2 (see label "Gaussian function"). The uncertainty with respect to this signal consists of:

- Uncertainty in location: the center of the signal $\mathbf{p}$ is unknown.

- Uncertainty in size: the scale parameter $\sigma$ is unknown.

- Uncertainty in orientation: the rotation angle $\vartheta$ is unknown.

- Uncertainty in signal contrast: the signal amplitude $a$ is unknown.

An interesting question that we will answer in the remaining of this section is: "How can a model observer deal with these sources of uncertainty?"

In the following, we will denote by $\alpha=\{\mathbf{p}, \sigma, \vartheta, a\}$ the vector of unknown signal parameters. We define $\mathbf{x}_{0}$ as the reference signal (i.e. a signal with the parameters $\alpha$ fixed to reference values). The signal vector $\mathbf{x}$ can further be transformed to the reference signal, using the linear transform $\mathbf{A}_{\alpha}: \mathbf{A}_{\alpha} \mathbf{x}_{\alpha}=\mathbf{x}_{0}$. 


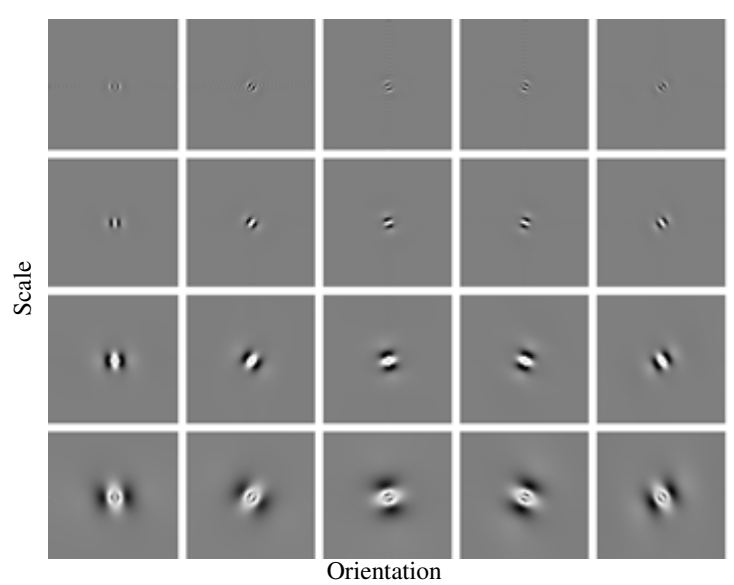

(a) Image domain

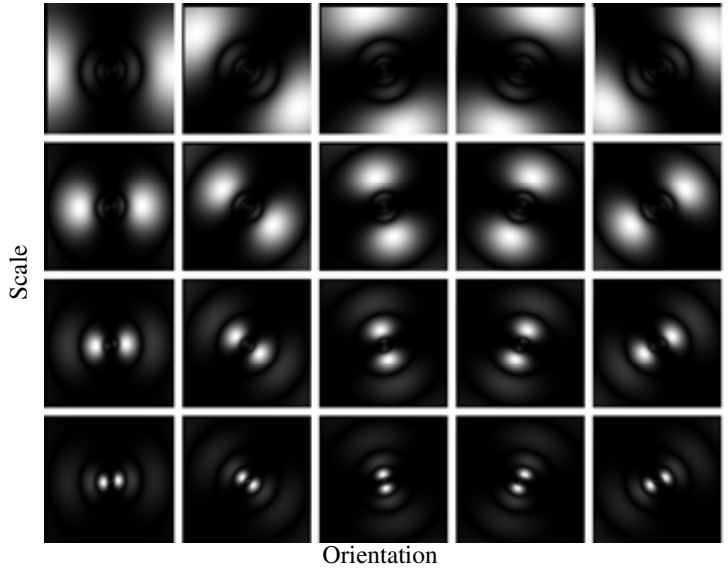

(b) Frequency domain

Figure 1. Example of channels that are jointly shiftable in scale and orientation. (a) Image domain (black corresponds to negative intensities, white to positive intensities, gray to zero), (b) Frequency domain (white corresponds to positive magnitude responses, black to zero magnitude responses).

The SKS detection task becomes an SKE task, when all of the above parameters are known exactly. It is also possible to design hybrid SKS/SKE detection tasks, in which some of the parameters are known, and other parameters are unknown. These detection tasks can all be performed using the CJO model. Remark that, the more parameters are unknown, generally the lower the detection performance will be: signals with more uncertainty are simply more difficult to detect. This generally holds both for model observers and for human observers.

In contrast to existing CHO models for SKS tasks (e.g., Ref $^{5}$ ), the CJO model jointly estimates the unknown signal parameters, together with the signal presence. This is done using joint detection and estimation theory. ${ }^{6}$ The CJO solves the following maximum a posteriori (MAP) problem:

$$
\left(\alpha, H_{k}\right)=\arg \max _{\left(\alpha, H_{k}\right)} f_{\alpha, H \mid \mathbf{y}}\left(\alpha, H_{k} \mid \mathbf{y}\right)
$$

where $f_{\alpha, H \mid \mathbf{y}}\left(\alpha, H_{k} \mid \mathbf{y}\right)$ is the posterior probability density function (pdf) of $\alpha, H$, given the observed signal vector. However, (4), is generally not trivial to solve:

1. It requires specification of the pdf $f_{\alpha, H \mid \mathbf{y}}\left(\alpha, H_{k} \mid \mathbf{y}\right)$, which depends on the conditional likelihood function $f_{\mathbf{y} \mid H}\left(\mathbf{y} \mid H_{k}\right)$. For complex medical images, as encountered in clinical practice, finding a good likelihood model is a very difficult task.

2. Maximizing $f_{\alpha, H \mid \mathbf{y}}\left(\alpha, H_{k} \mid \mathbf{y}\right)$ requires solving an optimization problem for which general techniques can be used. However, the dimensionality of the parameter space may be large, and the objective function may be non-convex.

To solve the first problem, the CJO uses a linear dimension reduction, by projecting onto a set of channels, like the CHO. To deal with the second problem, it was shown in $\operatorname{Ref}^{3}$ that evaluation of the likelihood function is substantially easier in channel space, when the channels $\mathbf{U}$ and the signal reference transform $\mathbf{A}_{\alpha}$ satisfy the following pseudo-commutative property:

$$
\mathbf{A}_{\alpha}^{T} \mathbf{U}=\mathbf{U A}_{\alpha}^{\prime T}
$$

The property can be interpreted as follows: a signal transform $\mathbf{A}_{\alpha}$ (such as rotation, scaling, translation) in the image space corresponds to an equivalent transform $\mathbf{A}_{\alpha}^{\prime}$ in channel space. In particular (5) imposes design constraints to the channel matrix $\mathbf{U}$, and it was shown that for the specific uncertainty sources we are considering, the following channel restrictions suffice $\left(\right.$ see $\left.\operatorname{Ref}^{3}\right)$ :

- Uncertainty in orientation: use steerable channels, similar to the steerable filters from Refs., ${ }^{7,8}$ 


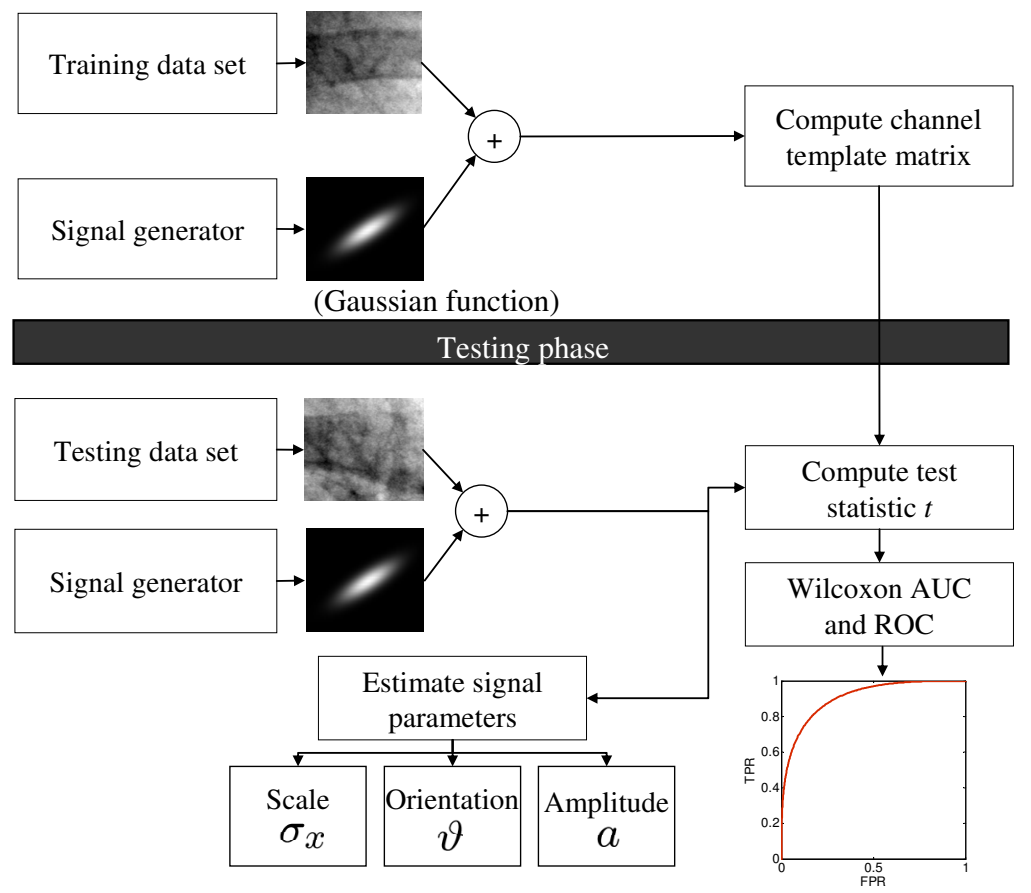

Figure 2. Overview of the channelized joint observer (CJO).

- Uncertainty in scale: use special scale-shiftable channels.

- Uncertainty in location: use a shift-invariant system, considering translates of the channel matrix.

- Uncertainty in signal contrast: no further constraints apply.

Moreover, when multiple sources of uncertainty occur simultaneously, the channel design constraints can be merged. For example, in the case of uncertainty in orientation/scale, this leads to channels that are steerable in orientation and shiftable in scale. An illustration of such channels is given in Figure 1.

The advantages of the CJO approach are:

1. The joint estimation and detection problem (4) is translated into a nearly (up to dimension reduction) equivalent problem in channel space. Because of the dimension reduction through channel projection, estimation of the unknown parameters and hence dealing with the uncertainty becomes much simpler.

2. The CJO signal detection and estimation consists of a non-linear estimation part and a linear detection part. Because of the linearity of the detection, linear discriminant analysis can still be used for model training. Consequently, the training and testing phases are very similar to the training and testing phases of the $\mathrm{CHO}\left(\mathrm{Ref}^{4}\right)$. See also the illustration in Figure 2.

In the following section, we will describe how the CJO can be used to estimate blur, noise and contrast of medical images.

\section{NOVEL APPROACH: CJO-BASED IMAGE QUALITY ASSESSMENT}

In this section, we present a novel image quality assessment approach that can be used for medical reconstruction techniques and/or medical displays. Here, we explicitly model the image degradation process (e.g., caused by acquisition) and the reconstruction process, but still consider both processes to be a black-box. Then, we intend to measure how the 


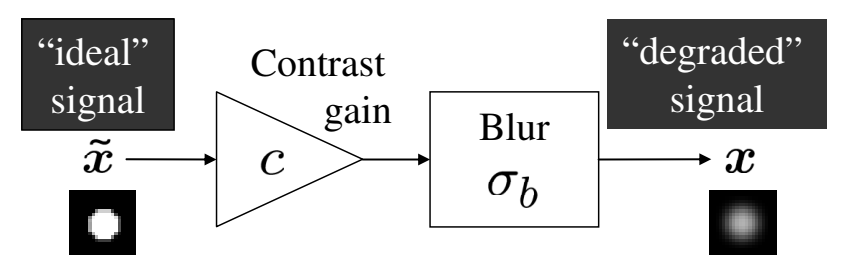

Figure 3. Illustration of the linear and space invariant (LSI) signal degradation process (T).

reconstruction process affects the quality of the reconstructed images, through signal insertion, with the goal to optimize the reconstruction parameters in terms of image quality. In the remainder of this paper, we will call the image, that is free from degradation and that we have not at our disposal, the ideal image. Furthermore, the image that is obtained after degradation and reconstruction will be referred to as the degraded image.

Suppose that the background and signal in (1) are given by:

$$
\begin{aligned}
\mathbf{b} & =\mathbf{f}_{\alpha, \beta}(\tilde{\mathbf{b}})+\mathbf{n}, \\
\mathbf{x}_{\alpha} & =\mathbf{T}_{\alpha} \tilde{\mathbf{x}}
\end{aligned}
$$

where $\tilde{\mathbf{b}}$ is an ideal background image (which we generally do not have at our disposal), $\tilde{\mathbf{x}}$ is the ideal (undegraded) signal and $\mathbf{n}$ is image noise. Note that $\tilde{\mathbf{x}}$ is not necessarily equal to the reference signal $\mathbf{x}_{0}$ from Section 2 (the ideal signal does not need to have fixed parameter values). The non-linear function $\mathbf{f}_{\alpha, \beta}(\cdot)$ models both the image degradation and reconstruction (in particular $\mathbf{f}_{\alpha, \beta}(\cdot)$ is considered to be a black box, hence unknown), where $\beta$ is the set of reconstruction parameters that we want to optimize. The linear transform $\mathbf{T}_{\alpha}$ (with $\alpha$ the set of degradation parameters) models the degradation of the "ideal" signal $\tilde{\mathbf{x}} .{ }^{\dagger}$ Next, we use a simplified degradation model for $\mathbf{T}_{\alpha}$ : we assume that $\mathbf{T}_{\alpha}$ consists of an intensity scaling (corresponding to signal contrast adjustment) and a Gaussian blurring operation (see Figure 3). This corresponds to a linear and space invariant (LSI) degradation process.

For a rotationally symmetric signal $\tilde{\mathbf{x}}$ from (2) with $b=1$, the degraded signal $\mathbf{x}_{\alpha}$ can be calculated analytically because the convolution of two Gaussian functions is again a Gaussian function:

$$
\left[\mathbf{x}_{\alpha}\right]_{\mathbf{q}}=\operatorname{ac} \exp \left(-\left(\mathbf{R}_{\vartheta} \mathbf{q}-\mathbf{p}\right)^{T} \mathbf{D}^{\prime-1}\left(\mathbf{R}_{\vartheta} \mathbf{q}-\mathbf{p}\right)\right) \quad \text { with } \quad \mathbf{D}^{\prime}=\left(\begin{array}{cc}
2\left(\sigma_{\tilde{\mathbf{x}}}^{2}+\sigma_{\text {blur }}^{2}\right) & 0 \\
0 & 2\left(\sigma_{\tilde{\mathbf{x}}}^{2}+\sigma_{\text {blur }}^{2}\right)
\end{array}\right) .
$$

Here, $\sigma_{\tilde{\mathbf{x}}}$ is the scale parameter of the undegraded signal $\tilde{\mathbf{x}}, \sigma_{\text {blur }}$ is the scale parameter of the Gaussian blur kernel and $c$ is the contrast change caused by the degradation. Hence, we are dealing with uncertainty in scale and uncertainty in signal contrast.

\subsection{Noise, contrast and blur}

Now, to estimate $\sigma_{\text {blur }}$ and $c$, the CJO can directly be used, as we will explain next. However, there is an important difference between our estimation approach discussed here and the related one in $\operatorname{Ref}^{3}$ : rather than estimating the degradation parameters $\sigma_{\text {blur }}$ and $c$ for each image individually, we will first compute the average signal and we then estimate the signal parameters. Because the initial averaging of the images reduces the noise, the signal parameters are estimated more accurately. This is particularly of importance when the noise variance $\operatorname{Var}[\mathbf{n}]$ is high.

Recall that the LSI degradation process modifies the parameters, according to equation (7). Taking advantage of the fact that the reconstruction process acts in the same way on all images (i.e., the image reconstruction itself is deterministic), we can improve the estimation performance: the degraded signal can be estimated simply by computing the difference between the signal-present images and the signal-absent images:

$$
\hat{\mathbf{x}}=\left\langle\mathbf{y} \mid H_{1}\right\rangle-\left\langle\mathbf{y} \mid H_{0}\right\rangle
$$

\footnotetext{
${ }^{\dagger}$ It can be checked that (6) is obtained by performing a first-order Taylor series approximation of $\mathbf{y}=\mathbf{f}_{\alpha, \beta}(\tilde{\mathbf{b}}+\tilde{\mathbf{x}})$ at $\tilde{\mathbf{x}}=\mathbf{0}$.
} 
where $\langle\cdot\rangle$ denotes the sample mean. Next, we estimate the signal parameters $\alpha=\left[c, \sigma_{x}\right]$ by least-squares fitting of (8) to (7):

$$
\widehat{\left(c, \sigma_{x}\right)}=\arg \min _{\left(c, \sigma_{x}\right)}\left\|\mathbf{x}\left(c, \sigma_{x}\right)-\hat{\mathbf{x}}\right\|^{2}=\arg \max _{\left(c, \sigma_{x}\right)} \mathbf{x}^{T}\left(c, \sigma_{x}\right)\left[\hat{\mathbf{x}}-\frac{1}{2} \mathbf{x}\left(c, \sigma_{x}\right)\right] .
$$

Note that the signal $\mathbf{x}\left(c, \sigma_{x}\right)$ depends on $\left(c, \sigma_{x}\right)$ in a non-linear way. To facilitate the estimation, we linearly project the original and estimated signals onto a set of scale-shiftable channels (see e.g. Figure 1):

$$
\mathbf{x}^{\prime}\left(c, \sigma_{x}\right)=\mathbf{U}^{T} \mathbf{x}\left(c, \sigma_{x}\right) \text { and } \hat{\mathbf{x}}^{\prime}=\mathbf{U}^{T} \hat{\mathbf{x}},
$$

where $\mathbf{U}$ is an $N \times K$ projection matrix containing $K$ column-stacked scale-shiftable channels (see Figure 1). Here, $N$ is the the number of pixels in the image (or length of the vector $\hat{\mathbf{x}}$ ). In channel space, (9) becomes:

$$
\widehat{\left(c, \sigma_{x}\right)}=\arg \max _{\left(c, \sigma_{x}\right)} \mathbf{x}^{T}\left(c, \sigma_{x}\right)\left[\hat{\mathbf{x}}^{\prime}-\frac{1}{2} \mathbf{x}^{\prime}\left(c, \sigma_{x}\right)\right] .
$$

Now, defining reference signal as $\mathbf{x}_{0}^{\prime}=\mathbf{x}^{\prime}(1,1)$, in the scale-shiftable channel domain, any signal $\mathbf{x}^{\prime}\left(c, \sigma_{x}\right)$ can be computed by applying a linear transform $\mathbf{A}_{c, \sigma_{x}}$ to the reference signal (see Section 2):

$$
\mathbf{x}^{\prime}\left(c, \sigma_{x}\right)=\mathbf{A}_{c, \sigma_{x}} \mathbf{x}_{0}^{\prime},
$$

where elements $m, n$ of $\mathbf{A}_{c, \sigma_{x}}$ are given by (see $\operatorname{Ref}^{3}$ ):

$$
\left[\mathbf{A}_{c, \sigma_{x}}\right]_{m n}=\frac{\sigma_{x}}{c} 2^{-(m-n)} \operatorname{sinc}\left(\log _{2} \sigma_{x}-(m-n)\right) .
$$

This gives the following maximization problem:

$$
\widehat{\left(c, \sigma_{x}\right)}=\arg \max _{\left(c, \sigma_{x}\right)} \mathbf{x}_{0}^{\prime T} \mathbf{A}_{c, \sigma_{x}}^{T}\left[\hat{\mathbf{x}}^{\prime}-\frac{1}{2} \mathbf{A}_{c, \sigma_{x}} \mathbf{x}_{0}^{\prime}\right] .
$$

The estimation can be further decoupled into explicit formulas for $\sigma_{b}$ and $c$. In particular, $\sigma_{x}$ can be optimally (in the sense of maximizing (14)) estimated using:

$$
\widehat{\sigma}_{x}=\arg \max _{\sigma_{x}} \mathbf{x}_{0}^{\prime T} \mathbf{A}_{1, \sigma_{x}}^{T}\left[\hat{\mathbf{x}}^{\prime}-\frac{1}{2} \mathbf{A}_{1, \sigma_{x}}^{T} \mathbf{x}_{0}^{\prime}\right] .
$$

Because the objective function in (15) is differentiable in $\sigma_{x}$, the maximum can easily be found using Gauss-Newton optimization techniques. This leads to the following estimate for the blur parameter:

$$
\widehat{\sigma_{b}}=\sqrt{\max \left(0, \widehat{\sigma}_{x}^{2}-\sigma_{\tilde{x}}^{2}\right)},
$$

where the maximum in the square root accounts for possible negative numbers due to estimation errors. Once the blur parameter is estimated, the contrast of the signal can be determined:

$$
\hat{c}=\frac{\mathbf{x}_{0}^{\prime T} \mathbf{A}_{1, \widehat{\sigma_{x}}}^{T} \hat{\mathbf{x}}^{\prime}}{\mathbf{x}_{0}^{\prime T} \mathbf{A}_{1, \widehat{\sigma_{x}}}^{T} \mathbf{A}_{1, \widehat{\sigma_{x}}} \mathbf{x}_{0}^{\prime}},
$$

which, given $\widehat{\sigma}_{x}$, is a linear function of $\hat{\mathbf{x}}^{\prime}$.

Now we turn to the estimation of the noise parameter $\sigma_{n}$. Using (1) and (6), we find that:

$$
\operatorname{Var}\left[\mathbf{y} \mid H_{0}\right]=\operatorname{Var}\left[\mathbf{y} \mid H_{1}\right]=\operatorname{Var}\left[\mathbf{f}_{\alpha, \beta}(\tilde{\mathbf{b}})\right]+\sigma_{n}^{2} \mathbf{I} .
$$

resulting in the following noise variance estimate:

$$
\widehat{\sigma_{n}^{2}}=\max \left(0, \frac{1}{N} \operatorname{trace}\left(\frac{1}{2}\left(\widehat{\operatorname{Var}\left[\mathbf{y} \mid H_{0}\right]}+\widehat{\operatorname{Var}\left[\mathbf{y} \mid H_{1}\right]}\right)-\widehat{\mathbf{C}}_{b}\right)\right),
$$

where $\widehat{\mathbf{C}_{b}}$ is an estimate of the ideal background variance $\mathbf{C}_{b}=\operatorname{Var}\left[\mathbf{f}_{\alpha, \beta}(\tilde{\mathbf{b}})\right]$. 


\subsection{Signal detectability}

The signal detectability can be estimated jointly with the signal parameters. However, as mentioned earlier, the estimation of the signal parameters is much more accurate when estimated on an ensemble of images, rather than on a per image basis. Correspondingly, there are two fundamentally different ways in evaluating the signal detectability:

1. Signal detectability in the SKE sense: in this case, we do not take the uncertainty with respect to the signal parameters (before reconstruction) into account.

Hence, the input signal parameter vector (before reconstruction) $\alpha_{\text {in }}=\left[a, \sigma_{\tilde{\mathbf{x}}}^{2}\right]$ is assumed to be known. We consider $\mathbf{x}\left(\hat{c}, \widehat{\sigma}_{x}\right)$ to be an accurate estimate of the signal $\mathbf{x}\left(c, \sigma_{x}\right)$, which is then used directly by the model observer. For example, for a simple matched filter (see $\operatorname{Ref}^{9}$ ), this gives:

$$
t=\left[\mathbf{x}\left(\hat{c}, \widehat{\sigma}_{x}\right)\right]^{T} \mathbf{y} .
$$

Alternatively, the $\mathrm{CHO}$ can be used, with test statistic:

$$
t=\left[\mathbf{x}^{\prime}\left(\hat{c}, \widehat{\sigma}_{x}\right)\right]^{T}\left(\mathbf{U}^{T}\left(\mathbf{C}_{b}+\sigma_{n}^{2} \mathbf{I}\right) \mathbf{U}\right)^{-1} \mathbf{U}^{T} \mathbf{y},
$$

where $\mathbf{C}_{b}+\sigma_{n}^{2} \mathbf{I}$ is then estimated in the training phase of the model. ${ }^{4}$ Next, AUC values can be calculated using the Mann-Whitney-Wilcoxon AUC test (see Refs ${ }^{4,10}$ ).

2. Signal detectability in the SKS sense: here, we assume that the signal parameter vector before reconstruction $\alpha_{\text {in }}=\left[a, \sigma_{\tilde{\mathbf{x}}}^{2}\right]$ is random, with a known probability density function. However, the signal parameter vector after reconstruction, $\alpha_{\text {out }}=\left[a c, \sigma_{x}^{2}\right]$ is also a random vector. Consequently, we need to re-estimate the signal parameters on a per image basis, because we cannot directly use (8) (which assumes that the input signal is fixed). Once the signal parameters are estimated, the test statistics $((19)$ and $(20))$ remain essentially the same.

For simplicity, we will use the matched filter (19) in the SKE sense to calculate the AUC for the results in this paper. An overview of this approach is given in Algorithm 1. Examination of the second approach will be a topic of our future research.

\section{EXPERIMENTAL RESULTS}

To assess the accuracy of the estimation approach discussed in Section 3, we extract 2000 patches of size $100 \times 100$ from the chest radiography database of Ref. ${ }^{11}$ We arranged them into 1000 signal-free patches, and 1000 patches with a signal inserted in the middle. The images are then artificially degraded using a Gaussian blur with parameter $\sigma_{b}$, and subsequently corrupted with noise with variance $\sigma_{n}^{2}$. The observation model is then given by:

$$
\mathbf{y}= \begin{cases}\mathbf{f}_{\alpha, \beta}(\tilde{\mathbf{b}})+\mathbf{n} & \left(H_{0}\right) \\ \mathbf{f}_{\alpha, \beta}(\tilde{\mathbf{b}})+\mathbf{n}+\mathbf{T} \tilde{\mathbf{x}} & \left(H_{1}\right)\end{cases}
$$

with $\mathbf{f}_{\alpha, \beta}(\tilde{\mathbf{b}})$ a Gaussian blurring operation (see Section 3). As an illustration, five signal present and five absent images are shown in Figure 4.

To jointly estimate the blur, contrast and AUC of the degraded images, we apply the blur estimation formula (15). Because the images are degraded using known blur levels and noise variances, we have ground-truth at our disposal to compare the estimation results to. The results are shown in Figure 5. It can be noted that the estimated quality metrics are quite accurate, with no noticeable influence of the backgrounds.

As a second experiment, we consider the evaluation of trajectories in MRI reconstruction. In this MRI experiment, raw data is acquired in the Fourier domain on non-Cartesian sampling points of a trajectory. A standard reconstruction technique for non-uniformly sampled MRI is the backward non-uniform Fourier transform (NUFT). The reconstruction is usually applied in an iterative way minimizing the $\ell_{2}$-norm (so-called least-squares NUFT). Unfortunately, this approach suffers from noise and streaking artifacts. ${ }^{12}$

To solve these problems, recently more advanced techniques have been proposed based on sparsity-based regularization, leading to non-linear optimization techniques. ${ }^{12,13}$ 
Algorithm 1 CJO-based image quality assessment of blur, noise, contrast and signal detectability.

1. For a generic signal profile $\mathbf{x}\left(c, \sigma_{x}\right)$, compute the reference signal $\mathbf{x}_{0}=\mathbf{x}(1,1)$.

2. Pre-compute the channel matrix $\mathbf{U}$.

3. Pre-compute the projected signal profile $\mathbf{x}_{0}^{\prime}=\mathbf{U}^{T} \mathbf{x}_{0}$.

4. Training phase (ideal images):

(a) Estimate the background covariance matrix $\mathbf{C}_{b}$ (note: this step can be skipped when using the matched filter, see Section 3).

5. Testing phase (degraded images):

(a) Project the input images $\mathbf{y}$ onto the channel matrix: $\mathbf{y}^{\prime}=\mathbf{U}^{T} \mathbf{y}$.

(b) Estimate the degraded signal $\hat{\mathbf{x}}^{\prime}=\left\langle\mathbf{y}^{\prime} \mid H_{1}\right\rangle-\left\langle\mathbf{y}^{\prime} \mid H_{0}\right\rangle$.

(c) Estimate the signal parameter $\widehat{\sigma}_{x}=\arg \max _{\sigma_{x}} \mathbf{x}_{0}^{\prime T} \mathbf{A}_{1, \sigma_{b}}^{T}\left[\hat{\mathbf{x}}^{\prime}-\frac{1}{2} \mathbf{A}_{1, \sigma_{b}}^{T} \mathbf{x}_{0}^{\prime}\right]$ with $\mathbf{A}_{1, \sigma_{b}}^{T}$ as defined by (13).

(d) Estimate the blur parameter $\widehat{\sigma_{b}}=\sqrt{\max \left(0, \widehat{\sigma}_{x}^{2}-\sigma_{\tilde{x}}^{2}\right)}$.

(e) Estimate the signal contrast $\hat{c}=\frac{\mathbf{x}_{0}^{\prime}{ }^{T} \mathbf{A}_{1, \widehat{\sigma_{x}}}^{T} \widehat{\mathbf{x}}^{\prime}}{\mathbf{x}_{0}{ }^{T} \mathbf{A}_{1, \widehat{\sigma_{x}}}^{T} \mathbf{A}_{1, \widehat{\sigma_{x}}} \mathbf{x}_{0}}$.

(f) Estimate the noise variance $\widehat{\sigma_{n}^{2}}=\max \left(0, \frac{1}{N} \operatorname{trace}\left(\frac{1}{2}\left(\widehat{\operatorname{Var}\left[\mathbf{y} \mid H_{0}\right]}+\widehat{\operatorname{Var}\left[\mathbf{y} \mid H_{1}\right]}\right)-\widehat{\mathbf{C}_{b}}\right)\right)$.

(g) Compute the test statistic on a per image basis, e.g., using the matched filter: $t=\left[\mathbf{x}\left(\hat{c}, \widehat{\sigma}_{x}\right)\right]^{T} \mathbf{y}$.

(h) Obtain the AUC value using the Mann-Whitney-Wilcoxon AUC test.
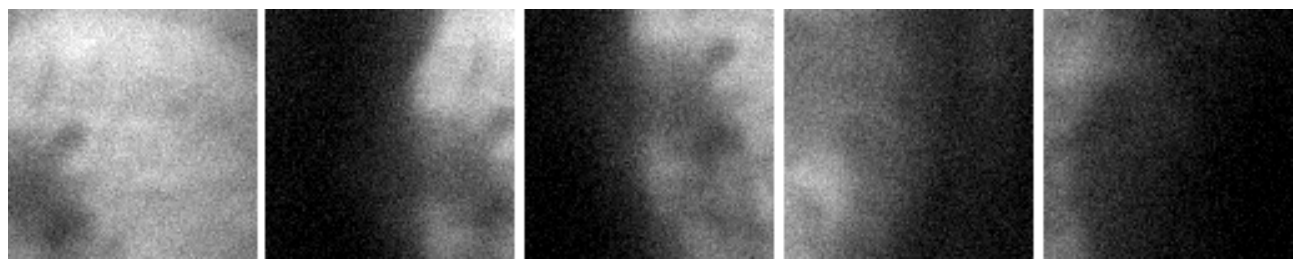

(a) Signal-free patches $\left(H_{0}\right)$
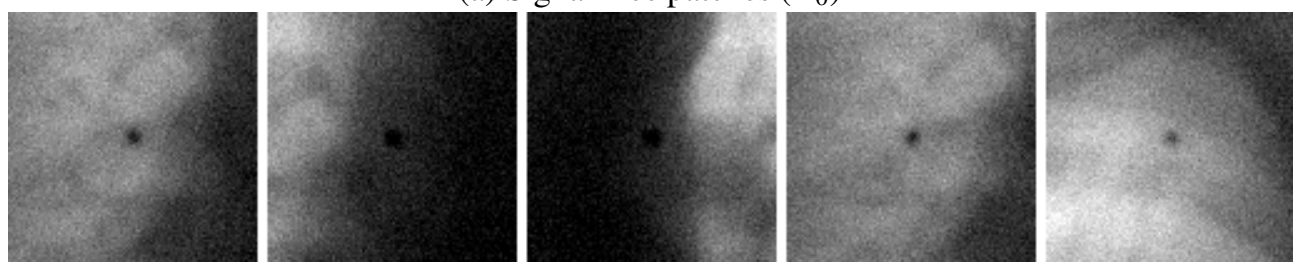

(b) Signal-present patches $\left(H_{1}\right)$

Figure 4. Degraded chest radiographs from the database from $\operatorname{Ref}^{11}\left(\sigma_{b}=2\right.$ and $\left.\sigma_{n}=5\right)$ (a) cropped patches, (b) cropped, with signal inserted in the middle. Note: the brightness and contrast of the images are enhanced for visualization purposes. 


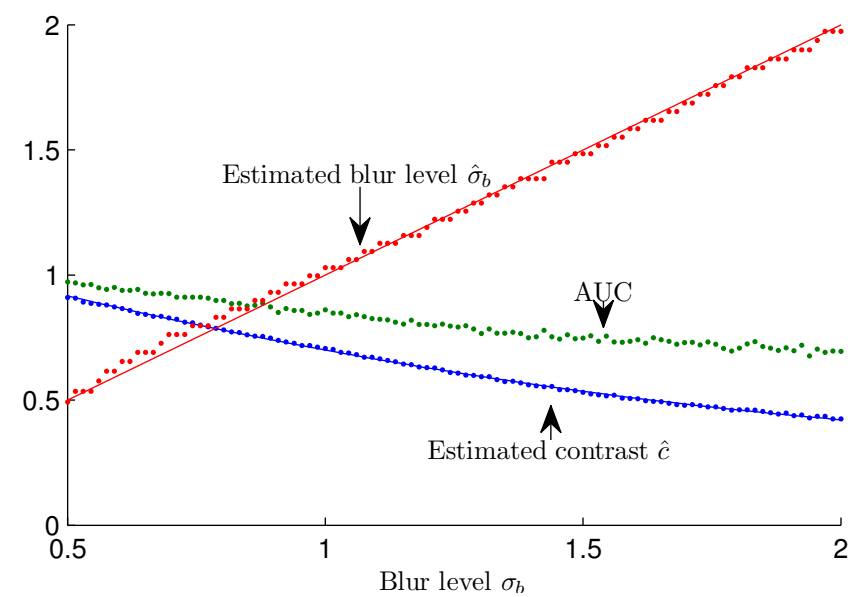

Figure 5. Plot of the estimated blur level vs. true blur level, estimated contrast vs. true contrast, and the estimated AUC.

Table 1. Quality measures for different frequency space trajectories, at 25\% of the Nyquist rate.

\begin{tabular}{c|cccc}
\hline Trajectory & RMSE & AUC & $\sigma_{b}$ & $c$ \\
\hline Cartesian sampling (center) & 7.66 & 0.79 & 0.47 & 0.97 \\
Radial sampling & 5.52 & 0.79 & 0.34 & 0.90 \\
Archimedean spiral & 4.39 & 0.79 & 0.30 & 0.91 \\
VD interleaved spiral & 4.40 & 0.80 & 0.30 & 0.92 \\
VD spiral (Ref ${ }^{1}$ ) & 7.36 & 0.78 & 0.30 & 0.87 \\
VD Poisson disk & 5.46 & 0.78 & 0.39 & 0.86 \\
\hline
\end{tabular}

Because the design space of spiral trajectories is huge, we evaluate the trajectories in a practical off-line procedure, using the proposed quality assessment (Section 3). First, we insert symmetrical Gaussian signals in the background of MRI images acquired at full Nyquist rate on a Cartesian grid. Subsequently, the MRI images are sub-sampled according to the considered spiral trajectories, at $25 \%$ of the Nyquist rate, and reconstructed using the method from Ref. ${ }^{12}$ Then 300 patches of size $32 \times 32$ are extracted out of the resulting images, of which 150 are signal-free and 150 are signal-present. Next, Algorithm 1 is applied to measure the blur parameter $\sigma_{b}$, signal contrast $c$ and the signal detectability (AUC). We also compute the root mean square error (RMSE), because MRI reconstruction techniques are often evaluated in terms of this metric.

The results are listed in Table 1. It can be noted that for all of the sampling schemes, the AUC is around 0.79 . However, the estimated blur and contrast levels vary a lot: the Archimedean spiral leads in this case to the lowest blur level, but also gives the lowest signal contrast. On the other hand, Cartesian sampling leads to the highest blur level (this is explained by the fact that only low-pass frequencies are being selected for sampling), but the signal contrast is the highest. It can also be noted that, as expected, AUC and RMSE values are completely unrelated. It is apparent that RMSE does not reflect well the diagnostic quality of the images. In particular, this is illustrated in Figure 6: in Figure 6(a) a lot of detail is lost compared to Figure 6(b), even though the RMSE values predict that Figure 6(a) has a better quality. For Figure 6(c) and (d), the signal detectability values (AUC) are equal, even though the blur level in Figure Figure 6(c) is higher than in Figure 6(d). Hence, the additional quality measures (blur and signal contrast) can help a lot for tuning the image reconstruction algorithm.

\section{CONCLUSION}

In medical image reconstruction, many trade-offs are possible between imaging and reconstruction parameters, while still yielding the same signal detectability in terms of AUC or detection SNR. In particular, resulting images with the same detectability, may still differ a lot in terms of blur and noise. Extra quality metrics allow to find a good trade-off between the parameters. In this paper, we extended the channelized joint observer (CJO) to estimate the signal blur, noise and contrast from degraded images. This approach allows us to estimate these quality metrics together with the signal detectability within the same space, obtained by projecting onto scale-shiftable channels. Our experimental results for MRI trajectory 


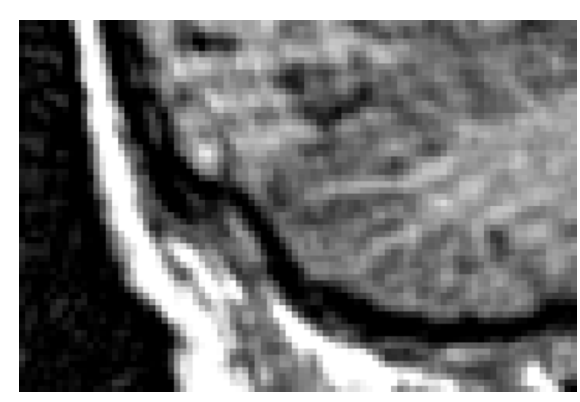

(a) $\mathrm{RMSE}=16.47, \mathrm{AUC}=0.69, \widehat{\sigma_{b}}=0.87, \hat{c}=0.66$

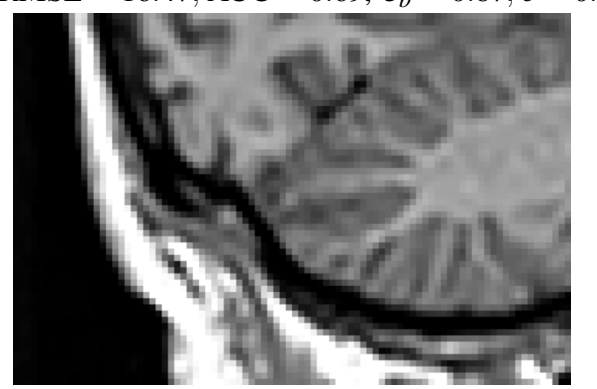

(c) $\mathrm{RMSE}=7.66, \mathrm{AUC}=0.79, \widehat{\sigma_{b}}=0.47, \hat{c}=0.97$

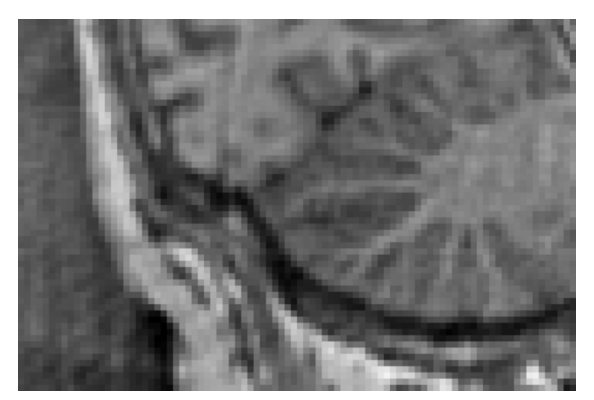

(b) $\mathrm{RMSE}=38.49, \mathrm{AUC}=0.77, \widehat{\sigma_{b}}=0.50, \hat{c}=0.45$

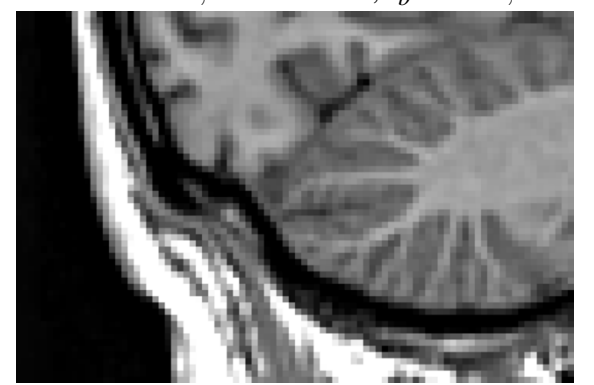

(d) $\mathrm{RMSE}=4.39, \mathrm{AUC}=0.79, \widehat{\sigma_{b}}=0.30, \hat{c}=0.91$

Figure 6. Visual MRI reconstruction results for different parameter values of the same trajectory.

optimization demonstrate that the proposed blur and contrast quality metrics help to compare the image quality of different images, even when the signal detectability is estimated to be equal. This result also indicates that a task-based approach for medical image quality assessment is very important. This paper provides thus a means to perform task-based image quality optimization.

\section{REFERENCES}

1. Lustig, M., Kim, S.-J., and Pauly, J., "A fast method for designing time-optimal gradient waveforms for arbitrary k-space trajectories.," IEEE Trans. Med. Imaging 27, 866-873 (June 2008).

2. Vandeghinste, B., Goossens, B., Van Holen, R., Vanhove, C., Pižurica, A., Vandenberghe, S., and Staelens, S., "Iterative CT reconstruction using shearlet-based regularization," in [SPIE Medical Imaging 2012], p. 83133I-(1-7) (Feb. 4-9 2012).

3. Goossens, B., Platiša, L., Vansteenkiste, E., and Philips, W., "Channelized Hotelling Observers for Detecting Signals with Random Parameters," In preparation. (2013).

4. Gallas, B. D. and Barrett, H. H., "Validating the use of channels to estimate the ideal linear observer," J. Opt. Soc. Am. A 20, 1725-1738 (sep 2003).

5. Park, S., Kupinski, M. A., Clarkson, E., and Barrett, H. H., "Ideal-observer performance under signal and background uncertainty," in [Information Processing in Medical Imaging], Taylor, C. J. and Noble, J. A., eds., 2732 in Lecture Notes in Computer Science, 342-353, Springer-Verlag, New York (2003).

6. Olmo, G., Magli, E., and Lo Presti, L., "Joint statistical signal detection and estimation. Part I: theoretical aspects of the problem," Signal Processing 80, 57-73 (jan 2000).

7. Freeman, W. and Adelson, E., "Design and use of steerable filters," IEEE Transactions on Pattern analysis and Machine Intelligence 13(9), 891-906 (1991).

8. Simoncelli, E. P. and Freeman, W. T., "The Steerable Pyramid: A flexible architecture for Multi-scale Derivative Computation," in [Proc IEEE Int.Conf. Image Processing], 444-447 (oct 1995).

9. Barrett, H. H., "Objective assessment of image quality: effects of quantum noise and object variability," J. Opt. Soc. Am. A 7, 1266-1278 (july 1990).

10. Wilcoxon, F., "Individual comparisons by ranking methods," Biometrics 1, 80-83 (1945). 
11. Shiraishi, J., Katsuragawa, S., Ikezoe, J., Matsumoto, T., Kobayashi, T., Komatsu, K., Matsui, M., Fujita, H., Kodera, Y., and Doi, K., "Development of a Digital Image Database for Chest Radiographs with and without a Lung Nodule: Receiver Operating Characteristic Analysis of Radiologists," Detection of Pulmonary Nodules, AJR 174, 71-74 (2000).

12. Aelterman, J., Luong, H., Goossens, B., Pižurica, A., and Philips, W., "Augmented Lagrangian based Reconstruction of non-uniformly sub-Nyquist sampled MRI data," Elsevier Signal Processing, Advances in Multirate Filter Bank Structures and Multiscale Representations 91(12), 2731-2742 (2011).

13. Lustig, M., Donoho, D., and Pauly, J., "Sparse MRI: The Application of Compressed Sensing for Rapid MR Imaging," Magnetic Resonance in Medicine 58, 1182-1195 (2007). 